\title{
Una propuesta de uso del Software de Geometría Dinámica como milieu para el diseño de una actividad aritmética: la división como un reparto equitativo
}

\author{
Julián Humberto Santos ${ }^{1}$
}

\begin{abstract}
Resumen
Este artículo presenta una idea de un diseño orientado desde la Teoría de las Situaciones Didácticas para niños de primer grado (5-6 años), que promueve el aprendizaje por adaptación en fases de trabajo adidáctico, y discusiones conjuntas en fases de trabajo con el grupo entero para movilizar colectivamente los conocimientos construidos por los estudiantes en las fases de trabajo adidáctico. El fin es atender a las nuevas exigencias de enseñanza y aprendizaje de las matemáticas a causa del confinamiento por la pandemia, principalmente la preocupación por el aprovechamiento del incremento del tiempo de trabajo individual de los estudiantes y la necesidad de optimizar el reducido tiempo de trabajo conjunto con el grupo entero y el profesor. Se usó el Software de Geometría Dinámica: DGPad-Colombia como un milieu con el cual los estudiantes interactúan en las fases de trabajo adidáctico para aprender. El objeto matemático que se pretende construir es la división como reparto equitativo; se entiende como reparto equitativo aquel que satisface a todos los participantes de la repartición. Este tipo de diseños son una alternativa para fortalecer los espacios de trabajo autónomo de los estudiantes sin ir en detrimento de los espacios de trabajo conjunto.
\end{abstract}

\section{Abstract}

This article presents an idea of a design oriented from the Theory of Didactic Situations for first graders (5-6 years old), which promotes learning by adaptation in phases of adidactic work, and joint discussions in phases of work with the whole group to collectively mobilize the knowledge constructed by the students in the phases of adidactic work. The purpose is to meet the new demands of teaching and learning mathematics due to the pandemic confinement, mainly the use of the increased individual work time of the students and the need to optimize the reduced joint work time with the whole group and the teacher. The Dynamic Geometry Software: DGPad-Colombia was used as a milieu with which the students interact in the phases of adidactic work to learn. The mathematical object to be constructed is the division as fair sharing; fair sharing is understood as that which satisfies all participants in the sharing. This type of design is an alternative to strengthen the students' autonomous workspaces without being detrimental to the joint workspaces.

\section{Palabras clave}

Software de Geometría Dinámica - DGPad-Colombia - Teoría de Situaciones Didácticas - División como reparto equitativo

\section{Keywords}

Dynamic Geometry Software - DGPad-Colombia - Theory of Didactic Situations - division as fair sharing

1 Universidad Distrital Francisco José de Caldas, Bogotá, Colombia

*Autor de correspondencia: jhsantost@correo.udistrital.edu.co

\section{Introducción}

A pocas semanas de haber comenzado el confinamiento a causa de la pandemia del COVID-19, me di cuenta de que el trabajo que solía tener en clase con mis estudiantes de primer grado (entre 5-6 años), usando materiales manipulativos que les permitieran interactuar para aprender resolviendo proble- 
mas, trabajando en pequeños grupos y luego discutiendo el trabajo hecho con la clase, parecía irreproducible en el espacio virtual.

El control del trabajo en pequeños grupos, sobre todo con niños tan pequeños, parecía difícil de ejercer; las herramientas que permitían espacios de reunión en pequeños grupos estaban fuera del alcance de la comprensión de los niños; se debía ahora consolidar un espacio de trabajo autónomo en el que los niños sin la compañía continua del profesor desarrollaran una actividad matemática, lo que reducía notoriamente las interacciones entre estudiantes y el profesor. Estos cambios podrían llevar a algunos profesores de matemáticas a proponer clases magistrales en la que se debilitaran las formas de interacción entre los estudiantes y que promovieran el aprendizaje como un proceso de imitación.

Para atender a esta coyuntura se pensó en diseños que permitieran responder a esta forma de gestionar el proceso de enseñanza y aprendizaje de las matemáticas promoviendo aprendizajes por adaptación. Se diseñaron actividades en las que se usó el Software de Geometría Dinámica: DGPadColombia como un milieu con el cual los estudiantes interactúan en los espacios de trabajo autónomo para aprender (Acosta, Blanco, y Gómez, 2010). El Software permite recuperar el trabajo individual de los estudiantes para que el profesor proponga un espacio de trabajo colectivo en donde se discuten los avances en la solución de las tareas, se comparten los conocimientos construidos y se promueven debates sobre los conocimientos construidos por otros estudiantes.

En este artículo se presenta uno de esos diseños, en los que se aprovecha las herramientas informáticas, y que tiene como objetivo recuperar el trabajo que se hacía en la clase regular para introducir de manera temprana la división en grado primero como un reparto, que es equitativo, cuando todos los sujetos que participan en el reparto están satisfechos. Se espera poder responder con este diseño a la pregunta ¿Cómo aprovechar el diseño de una actividad en el software para proponer una discusión colectiva en la que se recupere el trabajo autónomo de los estudiantes en las fases de trabajo adidáctico cuando se resuelve un problema de repartición?

\section{Orientaciones teóricas del diseño}

\subsection{La división como reparto equitativo en la clase de primer grado}

Algunos estudios dan cuenta de que los niños pueden enfrentarse a situaciones multiplicativas desde la educación primaria entre los 4 y 6 años; generalmente haciendo uso de estrategias indirectas (Bosch, Castro, y Segovia, 2007; Caballero, 2005; Castro y Hernández, 2014). Otros se centran por ejemplo en el aprendizaje informal de las operaciones multiplicación y división con niños entre los 4 y 7 años antes de comenzar con el estudio formal de las mismas (Ramírez y Castro, 2014). Según Rodríguez y cols. (2008), en general las investigaciones realizadas sobre las operaciones aritméticas elementales ponen de manifiesto que los niños antes de recibir enseñanza formal sobre los algoritmos, construyen por sí mismos un amplio abanico de estrategias.

Godino y Batanero al referirse a la progresión en el estudio de la división en niños de primaria mencionan que "en el caso de la división se trata de, repartir en partes iguales, hacer grupos iguales, restar reiteradamente, distribuir equitativamente, compartir, fraccionar, trocear, partir, etc." (Godino y Batanero, 2004, p. 210).

En relación con las estrategias utilizadas por los niños al resolver problemas donde se usan situaciones multiplicativas, autores como Wright, Mulligan, y Gould (2000) distinguen cinco niveles evolutivos en el conocimiento temprano de los niños sobre la multiplicación y la división: 1) agrupamiento inicial; 2) conteo perceptual por múltiplos; 3) comparación figurativa de grupos; 4) repetición abstracta de la composición de un grupo (por ejemplo, si se tienen seis grupos cada uno de tres elementos, uno de estos grupos es una unidad y al mismo tiempo tiene tres elementos), y; 5) multiplicación y división como operación.

Se hace necesario entonces reflexionar sobre la necesidad de que los niños aprendan a repartir en partes iguales antes de trabajar de forma directa con el objeto matemático como operación. Godino y Batanero afirman que en el caso de la división debemos tener en cuenta la existencia de dos sentidos distintos:

"resta sucesiva" de una cantidad fija d de otra D y lo que se debe hallar es el número de veces (q) que se puede restar hasta agotar D (la división como agrupamiento) o bien el sentido de "reparto en partes iguales" de una cantidad D entre un número dado de "sujetos" $d$, donde lo que se debe hallar es a cuánto tocan (q) (la división como distribución o reparto) (Godino y Batanero, 2004, p. 211).

En este diseño proponen situaciones en el sentido de la división como reparto en partes iguales. Aunque se reconoce que los niños se ven en la necesidad de hacer repartos en situaciones de su vida diaria con mucha frecuencia, es posible que no hayan discutido sobre la necesidad de considerar un reparto como una acción que satisface a todos los individuos que participan en esta. A este tipo de situaciones, en las que todos los sujetos que participan en el reparto están satisfechos, se les ha denominado situaciones de reparto equitativo. La estructura del diseño brinda la oportunidad de debatir con los estudiantes sobre como un reparto que satisface a todos es un reparto equitativo.

\subsection{La teoría de las Situaciones Didácticas}

La teoría de las Situaciones Didácticas (Brousseau, 2006) proporciona un marco sistémico para investigar los procesos de enseñanza y aprendizaje y también, para apoyar el diseño didáctico en las matemáticas. En la Teoría de Situaciones Didácticas el aprendizaje no es un proceso de transferencia simple, ni un proceso lineal y continuo. El aprendizaje se 
concibe como un proceso doble: un proceso de adaptación, asimilación y acomodación a un milieu que es productor de contradicciones, de dificultades y desequilibrios y, un proceso de aculturación, de adaptación a un saber cultural por la entrada en las prácticas de una institución (Houle, 2016; Mangiante-Orsola, Perrin-Glorian, y Strømskag, 2018; Margolinas y cols., 2011).

El aprendizaje por adaptación no contempla la intervención de un profesor. La teoría de Situaciones Didácticas considera la situación didáctica como aquella en la que se relacionan tres elementos: el profesor, el saber y el estudiante. Cuando el profesor tiene la intención de enseñar al estudiante un saber matemático dado se presenta una situación didáctica. Sin embargo, la Teoría de Situaciones Didácticas afirma que no es posible transmitir de manera directa el saber al estudiante y por lo tanto es necesaria una estrategia indirecta para la transmisión del saber. Esta estrategia indirecta consiste en crear las condiciones de un aprendizaje por adaptación, a través de lo que se llama una situación adidáctica. Una situación adidáctica es entonces aquella que privilegia la interacción del estudiante con un milieu para resolver un problema. Como el milieu es impersonal y no tiene ninguna intención didáctica de enseñar, a esa situación se le llama adidáctica.

El proceso de devolución centra su mirada en las acciones del profesor durante la situación adidáctica, examinando cómo acompaña el proceso de validación de sus estudiantes y cómo interviene para que el estudiante tenga un aprendizaje por adaptación. Teniendo en cuenta que la interacción de los estudiantes con el milieu en este diseño se dará sin la vigilancia del profesor, se han diseñado en el software retroacciones didácticas con el mismo objetivo de la devolución: reforzar el proceso de validación de los estudiantes evitando la interrupción de la interacción del sujeto con el milieu.

Según Artigue, Haspekian, y Corblin-Lenfant (2014) el milieu debe ser fuente de contradicciones y desequilibrios. Debe permitir que los estudiantes experimenten las limitaciones de sus estrategias iniciales, pero sus posibilidades de acción y retroalimentación también deben posibilitar una evolución hacia estrategias óptimas que den cuenta de la construcción de nuevos conocimientos.

Este diseño se basa en la imbricación del milieu con el Software de Geometría Dinámica propuesta por Acosta y cols. (2010) que permite a los estudiantes interactuar con el software para aprender por adaptación, el objetivo es entonces diseñar ese milieu que le permita a los estudiantes interactuar para aprender, pero que también le permita al profesor proponer una reflexión con el grupo de estudiantes en espacios que se han denominado puesta en común (Santos, 2016), mencionados por Margolinas y cols. (2011) como fases de balance, donde en un espacio dirigido y propuesto por el profesor, después de una fase adidáctica, se organiza un intercambio con todo el grupo, se discuten los avances y los conocimientos construidos.

\subsection{La puesta en común}

La puesta en común es un espacio colectivo que le permite al profesor proponer los conocimientos producidos por los estudiantes en las fases de trabajo adidáctico en el entorno de la clase, para avanzar en el proceso de construcción colectiva del saber. En la puesta en común se promueve el diálogo, las discusiones y los debates como caminos para impulsar nuevas formas de interacción que les permite a los estudiantes entender, por ejemplo, la responsabilidad que los hace participes de su aprendizaje y del aprendizaje de los otros (Romero y Gutiérrez, 2011).

Ya que en las actividades que se proponen en este diseño es débil el objetivo de abandonar estrategias por los estudiantes para resolver una tarea, en cambio es más fuerte el objetivo de enfrentarse a distintas formas de reparto y motivar una discusión posterior sobre esas situaciones y las implicaciones sociales que esto tiene, no es tan importante imponer un reparto correcto, se vuelve más importante reconocer cuál es un reparto que satisface a todos y cuál no.

En este sentido se debe reconocer en estos espacios que el conocimiento de la realidad se ha dado y se debe dar siempre a partir del otro y que esto implica un movimiento del yo hacia el otro, reconociendo las necesidades que tiene el otro y la necesidad de promover una nueva sensibilidad por el otro, que invierte nuestra noción de amor por la vida, desde el yo (como un ente ideal) a el otro (como un gozo al compartir). Esta noción de amor por la vida implica la posibilidad de acoger al otro (Lévinas, 2002).

Para esto en la fase de trabajo autónomo, se utiliza una representación de las emociones de las personas que están implicadas en la situación de reparto, esperando que al final se establezca con todo el grupo de estudiantes tras las discusiones en las puestas en común, que un reparto es equitativo cuando satisface a todos los individuos que participan en el reparto. Así el objetivo matemático que es introducir a los estudiantes en la división como un reparto equitativo queda supeditado a un objetivo sociocultural más amplio, generar acuerdos colectivos sobre un reparto equitativo.

\section{Secuencia de diseño}

Este diseño orientado teóricamente está previsto para ser implementado con niños de 4 a 6 años. Se han diseñado tres situaciones con diferentes tareas. Una vez terminada cada situación se espera que el profesor proponga una puesta en común en donde se discutirá con todo el grupo de estudiantes las acciones que cada uno consideró para solucionar cada una de las tareas.

El diseño se ha llevado a cabo con el Software de Geometría Dinámica DGPad-Colombia. Aunque el Software de Geometría Dinámica es un software diseñado para promover el aprendizaje de los objetos geométricos, se decidió explorar las bondades de este para producir un diseño que le permitiera a los niños aprender un objeto aritmético aprovechando algunas herramientas de su entorno, por ejemplo: la ubicación espacial de los puntos a partir de su sistema de coordenadas 
cartesianas que permitía ajustar los objetos de reparto para empezar una tarea nueva; o el conteo de objetos cuando estos están dentro o fuera de una circunferencia de radio fijo.

Está compuesto por botones de acción que los estudiantes deben presionar para comenzar y avanzar en el desarrollo de las tareas. Cada botón de acción que produce un mensaje en la pantalla está acompañado de un audio que lee el mensaje (previendo que algunos niños pueden no haber empezado su proceso de lectura). El software permite recuperar la forma como el estudiante lleva a cabo los repartos, información que le permitirá al profesor planificar las discusiones en las puestas en común sobre las estrategias usadas por los estudiantes.

A continuación, se presenta cada una de las situaciones, se describe el objetivo esperado, un modelo de las tareas propuestas en cada situación y las acciones esperadas por parte de los estudiantes, teniendo en cuenta que estas acciones no son necesariamente consecutivas, con las retroacciones didácticas para cada acción. Luego se describirán las discusiones que se esperan por parte del profesor en la puesta en común.

\subsection{Situación 1}

Tú has invitado a tres amigos a la celebración de tus cumpleaños: Julián, Camilo y Mario. Tu papá te ha pedido ayudarle a repartir los dulces y juguetes en las 4 bolsas de sorpresas (ver Figura 1).

\section{Objetivos}

1. Identificar si el estudiante dota a la acción de repartir de una necesidad de repartir en partes iguales para todos los sujetos que participan en la repartición

2. Discutir sobre la idea de repartir en partes iguales como un reparto equitativo

Tareas Para esta situación se diseñaron cinco tareas en las cuales luego de cada reparto no sobraban objetos sin repartir. Una de esas tareas era: Reparte 20 silbatos en las 4 bolsas de sorpresas.

\section{Acciones y retroacciones didácticas}

Acción 1: el estudiante no hace ningún reparto y oprime el botón tarea terminada. Retroacción didáctica: el software reacciona con un letrero que informa que aún no se ha empezado el reparto.

Acción 2: el estudiante deja objetos sin repartir y oprime el botón tarea terminada. Retroacción didáctica: el software reacciona con un letrero que informa que aún faltan objetos por repartir.

Acción 3: el estudiante reparte los silbatos en las bolsas colocando más silbatos en la bolsa de él y oprime el botón tarea terminada. Retroacción didáctica: los personajes Julián, Camilo y Mario reaccionan preguntando por qué han recibido menos (aparecen imágenes tristes que representan a los personajes), luego lo invita a continuar con la tarea dos.
Acción 4: el estudiante reparte los silbatos en las bolsas colocando más silbatos en la bolsa de Julián, Camilo o Mario y oprime el botón tarea terminada. Retroacción didáctica: el software reacciona con un letrero que informa que ha colocado menos objetos en la bolsa de él (aparece una imagen inconforme que representa al personaje), luego lo invita a continuar con la tarea dos.

Acción 5: el estudiante reparte los silbatos en las bolsas colocando la misma cantidad de silbatos en las cuatro bolsas y oprime el botón tarea terminada. Retroacción didáctica: el software reacciona con un letrero que informa que ha repartido los silbatos y todos han recibido lo mismo (aparecen imágenes felices que representan a los personajes), luego lo invita a continuar con la tarea dos.

Puesta en común El profesor debe proponer una puesta en común luego del trabajo autónomo de los estudiantes con el software en el desarrollo de la situación 1. El profesor después de recuperar el trabajo de los estudiantes en la fase de trabajo autónomo, debe empezar por mostrar al grupo un registro del trabajo de los estudiantes que reparten los silbatos en las bolsas colocando más silbatos en la bolsa de él, para abrir el debate sobre por qué se eligió esta acción.

Los estudiantes podrían manifestar que en su repartición pensaron en tomar más para él porque era su fiesta; tomar más para él porque si él recibe más, él está más feliz; o que la tarea no pedía hacer una repartición por partes iguales.

El profesor debe proponer a este grupo de estudiantes preguntas cómo ¿Cuál era la expresión de los personajes cuando recibían menos que él? ¿Cómo se sentirían los demás si al abrir una bolsa de sorpresas tuvieran menos que los otros? ¿Cómo se sentiría cada uno si tuviera menos que el otro?

El profesor debe entonces mostrar al grupo un registro del trabajo de los estudiantes que reparten los silbatos en las bolsas colocando menos silbatos en la bolsa de él, para abrir el debate sobre por qué se eligió esta acción. Los estudiantes podrían manifestar que en su repartición pensaron en tomar menos objetos en su bolsa para satisfacer a sus amigos o que la tarea no pedía hacer una repartición por partes iguales. El profesor puede recurrir de nuevo a discutir sobre las expresiones de personajes cuando esto pasaba, podría proponer preguntas similares a las propuestas en la discusión anterior.

Para finalizar, el profesor debe mostrar al grupo un registro del trabajo de los estudiantes que reparten los silbatos en las bolsas colocando la misma cantidad de silbatos en cada bolsa, para abrir el debate sobre por qué se eligió esta acción.

Los estudiantes podrían manifestar que pensaron en un reparto que satisface a todos donde todos reciben lo mismo, nadie más o menos que otro, un reparto con el que todos son felices. El profesor debe proponer al grupo de estudiantes preguntas cómo ¿Cuál era la expresión de los personajes cuando recibían lo mismo? ¿Qué tan importante es que, al abrir las bolsas de sorpresa en la fiesta, cada uno tengo la misma cantidad de juguetes? 


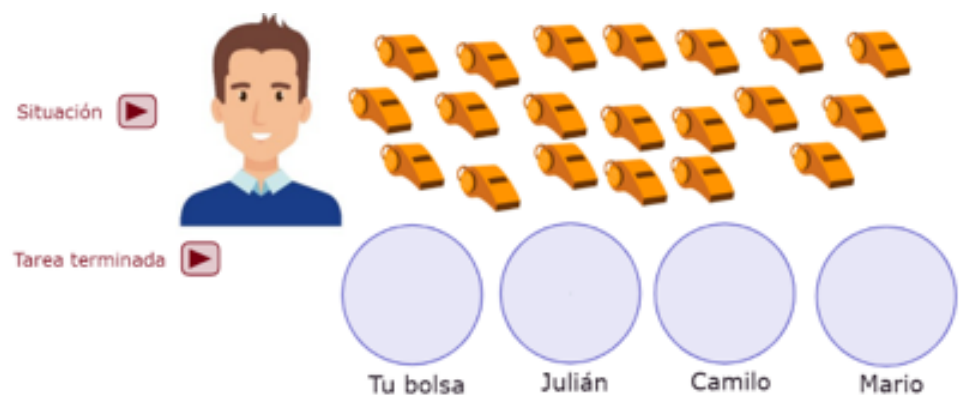

Reiniciar tarea

Vuelve a empezar

Figura 1. Situación 1, tarea 1

Se espera que las discusiones del grupo permitan generar un acuerdo colectivo sobre: una repartición que satisface a todos es una repartición en la que cada uno recibe la misma cantidad.

\subsection{Situación 2}

Tú has invitado a tres amigos a la celebración de tus cumpleaños: Julián, Camilo y Mario. Tu papá te ha pedido ayudarle a repartir los dulces y juguetes en las 4 bolsas de sorpresas.

\section{Objetivos}

1. Identificar si el estudiante reconoce que una repartición es equitativa cuando cada uno recibe lo mismo sin importar que sobren objetos sin repartir

Tareas Para esta situación se diseñaron cinco tareas en las cuales luego de cada reparto equitativo sobraban objetos $\sin$ repartir. Una de esas tareas era: Reparte 18 dulces en las 4 bolsas de sorpresas (ver Figura 2).

Acciones y retroacciones didácticas Se espera que se lleven a cabo acciones similares a las descritas en la situación anterior, con la variante de tener que controlar a quién colocar los objetos que sobran. En este sentido no se mencionarán de nuevo las acciones de 1 a 4 descritas anteriormente.

Acción 5: el estudiante reparte el máximo de dulces posibles en las bolsas colocando la misma cantidad en cada una, dejando los dulces sobrantes fuera de la repartición y presiona el botón tarea terminada. Retroacción didáctica: el software reacciona con un letrero que informa que ha repartido los dulces y todos han recibido lo mismo, luego lo invita a continuar con la tarea siguiente.

Puesta en común La discusión que debe proponer el profesor en esta puesta en común debe girar en torno a cómo se repartieron los objetos que sobran. El profesor después de recuperar el trabajo de los estudiantes en la fase de trabajo autónomo, debe mostrar al grupo un registro del trabajo de los estudiantes que colocaban el objeto que sobraba en su bolsa o en la bolsa de otro, para preguntar sobre el porqué de sus acciones.
Se espera que los estudiantes manifiesten tener que repartir ese objeto, porque esa era la instrucción de la tarea. El profesor puede preguntar de nuevo si esta sería una repartición en la que todos recibirían lo mismo.

El profesor puede proponer a estos estudiantes preguntas cómo ¿Cuál era la expresión de los personajes cuando recibían menos que el otro? ¿Qué tan importante es que, al abrir las bolsas de sorpresa en la fiesta, cada uno tengo la misma cantidad de juguetes?

Una discusión que podría darse en la puesta en común es que podría pensarse en partir los objetos sobrantes para que cada niño reciba lo mismo. El profesor debe entonces discutir con los estudiantes sobre la naturaleza de los objetos ¿Cómo partir un silbato en dos partes iguales? ¿Seguiría siendo un silbato?

El profesor debe mostrar la importancia de que en algunas reparticiones en las que todos reciben lo mismo, puede haber objetos que sobran y que no se pueden partir. Habrá objetos que no podrán ser partidos porque dejarían de ser útiles (una bola).

Otra discusión que podría surgir es, en un contexto cotidiano, qué hacer con el objeto que sobra, quién se debe quedar con él. Esta sería una gran oportunidad para reflexionar sobre otros tipos de repartos que podrían darse en la vida diaria, quién estaría a dispuesto a ceder sobre ese objeto, quién debería por derecho o acuerdo tomarlo, deberíamos devolverlo, no deberíamos tenerlo en cuenta.

Se espera que las discusiones del grupo permitan generar un acuerdo colectivo sobre: una repartición satisface a todos cuando se reparte el máximo de objetos posibles y cada uno recibe la misma cantidad, así queden objetos sin ser repartidos.

\subsection{Situación 3}

Tu profesor ha hecho unas reparticiones para algunos estudiantes de la clase ¿Te parece que la repartición satisface a todos? (ver Figura 3). Esta situación no está acompañada de una puesta en común, se diseñó como una situación de evaluación.

\section{Objetivos}




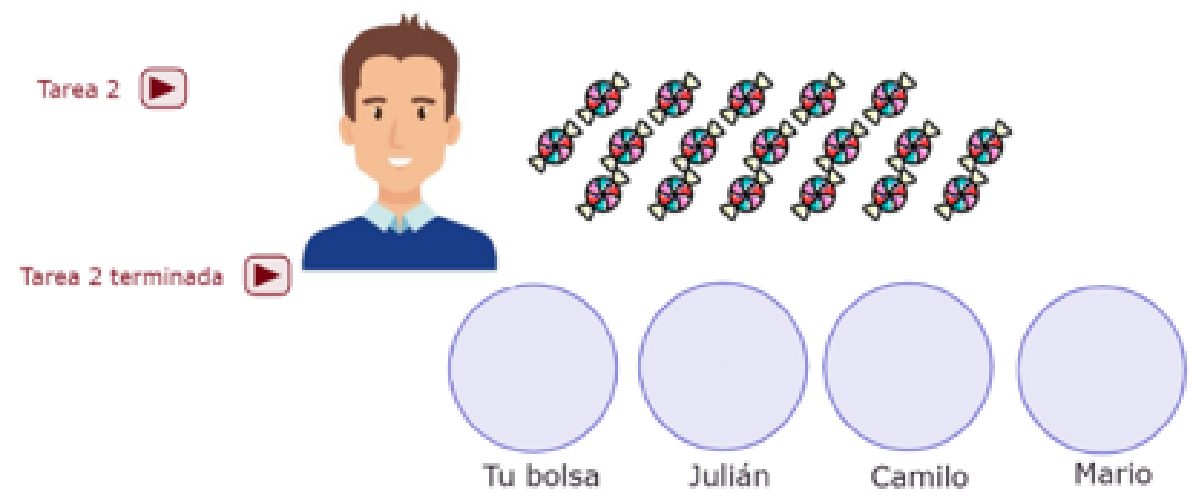

Reiniciar tarea

Figura 2. Situación 2, tarea 2

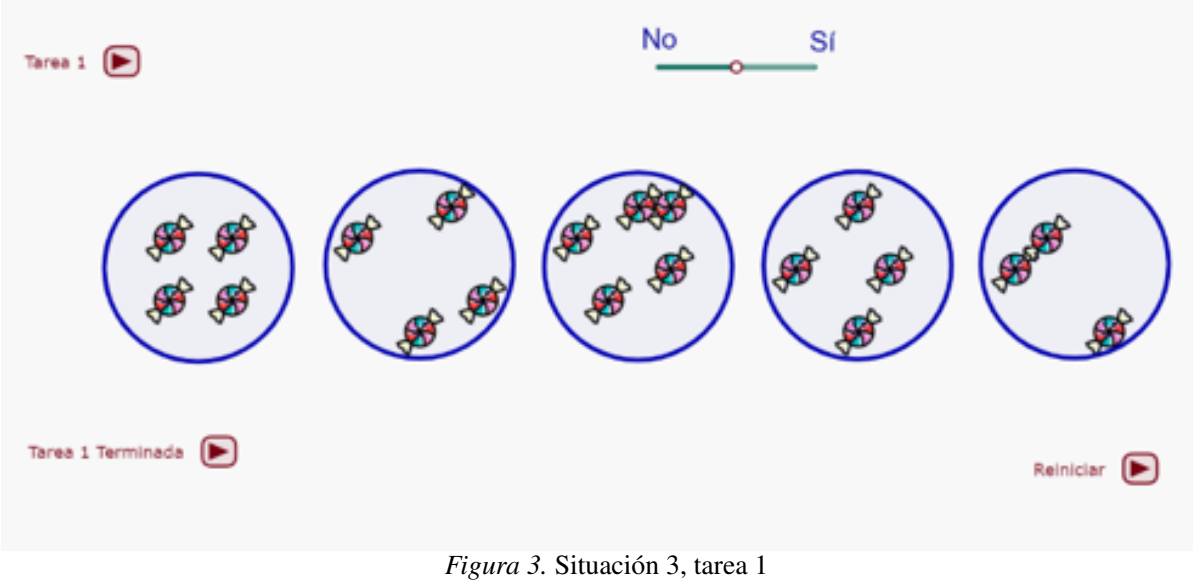

1. Evaluar los repartos de los estudiantes luego de las fases de trabajo adidáctico y puestas en común.

Tareas Para esta situación se diseñaron ocho tareas en las cuales aparecían algunos repartos ya establecidos y el estudiante debía tomar una postura sobre si este, satisface o no a todos. En estas tareas no solo variaba el número de objetos, también el número de bolsas.

\section{Acciones y retroacciones didácticas}

Acción 1: el estudiante selecciona la opción de sí hay un reparto que satisface a todos, aunque no todas las bolsas tienen la misma cantidad de objetos y presiona el botón tarea terminada. Retroacción didáctica: el software reacciona con un letrero que le pregunta ¿Cuántos dulces hay en cada bolsa?

Acción 2: el estudiante selecciona la opción de no hay un reparto que satisface a todos, reconociendo que no todas las bolsas tienen la misma cantidad de objetos y presiona el botón tarea terminada. Retroacción didáctica: el software reacciona con un letrero que le pregunta
¿Puedes redistribuir los dulces para tener un reparto que satisface a todos?

Acción 3: el estudiante redistribuye los dulces, pero no logra que todas las bolsas tengan la misma cantidad de objetos y presiona el botón tarea terminada. Retroacción didáctica: el software reacciona con un letrero que le pregunta ¿Cuántos dulces hay en cada bolsa? luego lo invita a continuar con la tarea siguiente.

Acción 4: el estudiante redistribuye los dulces logrando que todas las bolsas tengan la misma cantidad de objetos y presiona el botón tarea terminada. Devolución: Retroacción didáctica: el software reacciona con un letrero que informa que ha repartido los dulces y todos han recibido lo mismo, luego lo invita a continuar con la tarea siguiente.

\section{Conclusiones}

El diseño aquí presentado permite recuperar las estrategias de los repartos hechas por los estudiantes en una fase de trabajo adidáctico, para que luego el profesor pueda proponer 
en base a estas una discusión conjunta en un espacio que se ha denominado puesta en común. Esto le permite al profesor gestionar una clase no magistral que promueva el debate sobre las acciones que el otro lleva a cabo para resolver una tarea.

Es posible pensar que el diseño de una actividad usando tecnologías, en este caso el Software de Geometría Dinámica, permite proponer una discusión conjunta en la que se generen acuerdos colectivos sobre producciones personales de los estudiantes, que luego de las interacciones con el milieu en las fases de trabajo adidáctico, promovidas esencialmente por las retroacciones didácticas diseñadas por el profesor, comparten sus conocimientos con la clase.

Así este diseño permitió atender a las nuevas exigencias de enseñanza y aprendizaje de las matemáticas a causa del confinamiento por la pandemia, aprovechando el notorio incremento del tiempo de trabajo individual de los estudiantes y evitando la acción de proponer clases magistrales en la que se debilitaran las formas de interacción entre los estudiantes promoviendo un aprendizaje por imitación.

Aunque algunos autores han estudiado las ventajas del uso del Software de Geometría Dinámica para la enseñanza de las matemáticas (Leung y Lopez-Real, 2002; Mariotti, 2002; Marrades y Gutiérrez, 2000), este diseño presenta el potencial que puede tener el Software DGPad-Colombia cuando se usa como un medio aritmético que permite la interacción de los estudiantes para aprender, especialmente por la oportunidad de diseñar retroacciones didácticas que permiten reforzar el proceso de validación de los estudiantes y recuperar las estrategias que los estudiantes usan para solucionar una tarea.

El diseño permite que los niños se enfrenten a situaciones de reparto para construir uno de los dos sentidos de la división propuestos por Godino y Batanero (2004), antes de recibir enseñanza formal sobre los algoritmos, el "reparto en partes iguales" a partir de un acuerdo colectivo: una repartición que satisface a todos es una repartición en la que cada uno recibe la misma cantidad.

\section{Agradecimientos}

El diseño presentado en este artículo fue producto de la revisión teórica y metodológica explorada en el marco del Doctorado Interinstitucional en Educación de la Universidad Distrital Francisco José de Caldas en Bogotá, Colombia. Extiendo mi más fraterno agradecimiento a mi tutor el Dr. Martín Acosta por su importante aporte a la concreción de las ideas aquí presentadas.

\section{Referencias}

Acosta, M., Blanco, L., y Gómez, K. (2010). Situaciones a-didácticas para la enseñanza de la simetría axial utilizando cabri como medio. Revista Integración, 28(2), 173-189.

Artigue, M., Haspekian, M., y Corblin-Lenfant, A. (2014). Introduction to the theory of didactical situations (tsd.
En Networking of theories as a research practice in mathematics education (p. 47-65). Cham: Springer.

Bosch, A., Castro, E., y Segovia, I. (2007). El pensamiento multiplicativo en los primeros niveles: una investigación en curso. PNA, 1(4), 170-190.

Brousseau, G. (2006). Theory of didactical situations in mathematics: Didactique des mathématiques, 1970-1990. Springer Science \& Business Media.

Caballero, S. (2005). Un estudio transversal y longitudinal sobre los conocimientos informales de las operaciones aritméticas básicas en niños de educación infantil [tesis doctoral. Universidad Complutense de Madrid, España.

Castro, C., y Hernández, E. (2014). Problemas verbales de descomposición multiplicativa de cantidades en educación infantil. PNA, 8(3), 99-114.

Godino, J., y Batanero, C. (2004). Didáctica de las matemáticas para maestros.

Houle, V. (2016). Étude de conditions didactiques favorables À la décontextualisation des connaissances mathématiques. Canadian Journal of Education, 39(4), 1-19.

Leung, A., y Lopez-Real, F. (2002). Theorem justification and acquisition in dynamic geometry: a case of proof by contradiction. International Journal of Computers for Mathematical Learning, 7(2), 145-165.

Lévinas, E. (2002). Totalidad e infinito: ensayo sobre la exterioridad (Sexta ed.). Salamanca: Ediciones.

Mangiante-Orsola, C., Perrin-Glorian, M.-J., y Strømskag, H. (2018). Theory of didactical situations as a tool to understand and develop mathematics teaching practices. En Annales de didactique et de sciences cognitives (p. 145-174).

Margolinas, C., Abboud-Blanchard, M., Bueno-Ravel, L., Douek, N., Fluckiger, A., y Gibel, P. (2011). En amont et en aval des ingénieries didactiques. La pensée sauvage.

Mariotti, M. (2002). Justifying and proving in the cabri environment. International Journal of Computers for Mathematical Learning, 6(3), 257-281.

Marrades, R., y Gutiérrez, A. (2000). Proofs produced by secondary school students learning geometry in a dynamic computer environment. Educational studies in mathematics, 44(1-2), 87-125.

Ramírez, M., y Castro, C. (2014). Comprensión de las decenas y aplicabilidad de las operaciones en problemas aritméticos verbales. Investigación en Educación Matemática, XVIII, 533-542.

Rodríguez, P., Lago, M., Caballero, S., Dopico, C., Jiménez, L., y Solbes, I. (2008). El desarrollo de las estrategias infantiles. (Un estudio sobre el razonamiento aditivo y multiplicativo. Anales de Psicología/Annals of Psychology, 24(2), )

Romero, E., y Gutiérrez, M. (2011). La idea de responsabilidad en lévinas: implicaciones educativas. En Xii congreso internacional de teoría de la educación (p. 1-12). 
Universitat de Barcelona.

Santos, J. (2016). Una implementación para la construcción del objeto geométrico parábola, a partir del.

Wright, J., Mulligan, J., y Gould, P. (2000). Extending the learning framework to multiplication and division. Assessment for teaching and intervention, 154-176. 